\title{
A lepto-hadronic model of gamma-ray signal from extreme blazars
}

\author{
Saikat Das, ${ }^{a, *}$ Nayantara Gupta ${ }^{a}$ and Soebur Razzaque ${ }^{b}$ \\ ${ }^{a}$ Astronomy \& Astrophysics Group, Raman Research Institute, Bengaluru 560080, Karnataka, India \\ ${ }^{b}$ Centre for Astro-Particle Physics (CAPP) and Department of Physics, University of Johannesburg, P.O. \\ Box 524, Auckland Park 2006, South Africa \\ E-mail: saikatdas@rri.res.in
}

\begin{abstract}
The unattenuated $\mathrm{TeV} \gamma$-ray spectrum observed in some blazars is inconsistent with the $\gamma \gamma$ absorption by seed photons in the relativistic jet or the extragalactic background light (EBL) during extragalactic propagation. In addition, the efficiency of inverse-Compton emission is suppressed at such high energies. Ultrahigh-energy cosmic rays (UHECRs; $E \gtrsim 10^{17} \mathrm{eV}$ ) accelerated in blazar jets can escape from their sources and interact with the cosmic background photons. The resultant $\mathrm{e}^{ \pm}$and $\gamma$-rays can induce electromagnetic cascade resulting in a photon spectrum peaking at $\sim 1$ $\mathrm{TeV}$ energies. We propose that the line-of-sight component can be observed as coming from their respective sources. We consider a random turbulent extragalactic magnetic field to constrain the survival fraction of UHECRs along the observer's line of sight. A one-zone leptonic model is used to fit the synchrotron spectrum of the blazars. The higher-energy peak is explained by a combination of synchrotron self-Compton spectrum produced by the same relativistic electrons and the $\gamma$-rays from line-of-sight UHECR interactions. Overall, the lepto-hadronic model better explains the multiwavelength spectral energy distribution (SED). We also calculate the line-ofsight neutrino flux from these blazars corresponding to the luminosity required in $\gamma$-rays. We discuss the prospects of UHECR detection from these resolved sources.
\end{abstract}

$37^{\text {th }}$ International Cosmic Ray Conference (ICRC 2021)

July 12th-23rd, 2021

Online - Berlin, Germany

\footnotetext{
${ }^{*}$ Presenter
} 


\section{Introduction}

Active galactic nuclei (AGNs) are potential candidates of ultrahigh-energy cosmic-ray (UHECR; $E \gtrsim 10^{17} \mathrm{eV}$ ) accelerators. The jetted outflow of AGNs contains a tangled magnetic field and a relativistic population of electrons and protons, accelerated by the same inherent mechanism. Blazars, a subclass of radio-loud AGNs, have their jets pointed towards the Earth. Their spectral energy distribution (SED) has a low-energy peak between optical to X-ray energies and a high-energy peak at the $\gamma$-ray regime. The low-energy peak is almost evidently from leptonic synchrotron emission. The high-energy peak can be due to inverse-Compton scattering of synchrotron or external photons, such as from broad-line region, dusty torus, and accretion disk, by relativistic electrons. Alternatively, cosmic-ray interactions with photons inside the jet can produce secondary electrons and $\gamma$-rays. These $\gamma$-rays and the synchrotron emission of secondary electrons can produce the high-energy peak. The total luminosity budget must be within the Eddington limit of the source [1]. High-frequency peaked BL Lacs (HBLs) have their peak synchrotron emission in the UV to X-ray energy range, and their spectrum extends up to multi-TeV energies. High-energy photons inside the jet are susceptible to absorption by intrinsic $\gamma \gamma$ pair production or the extragalactic background light (EBL) while propagating over cosmological distances. Hence the intrinsic spectrum must be harder than the observed spectrum.

For some HBLs, the observed spectrum at $\mathrm{TeV}$ energies is too hard, contrary to the suppression due to absorption or Klein-Nishina effect. We explain the $\mathrm{TeV}$ spectra by the line-of-sight interaction of UHECRs with the cosmic background photons, producing secondary $\mathrm{e}^{ \pm}$and $\gamma$-rays $[2,3]$. They induce electromagnetic (EM) cascade and produce a resultant $\gamma$-ray spectra at very-high energies (VHE; $E>30 \mathrm{GeV}$ ). The threshold energy of protons for photopion production with the cosmic microwave background $(\mathrm{CMB})$ is $E \geqslant 50 \mathrm{EeV}$. At lower energies, interactions with the EBL photon dominates. We consider a few high-energy BL Lacs with suitable parameters for UHECR acceleration. We model the multiwavelength SED in the quiescent state by a leptonic synchrotron/SSC model and also using a lepto-hadronic model where the VHE photons originate from UHECR interactions close to the line of sight. The lepto-hadronic model explains better the VHE $\gamma$-ray data. UHECR protons are deflected by extragalactic magnetic fields (EGMF). We constrain the EGMF such that a significant fraction of UHECR protons are constricted near the line of sight, respecting the luminosity budget. We calculate the required kinetic power in electrons and protons to compare it with the Eddington luminosity of the AGN.

\section{Leptonic \& Hadronic Modeling}

The emission region is a spherical blob of radius $R$ contains a relativistic plasma of $\mathrm{e}^{-}$and $p$ in a magnetic field $B$. The constant electron injection in the jet comoving frame is $Q_{e}\left(E_{e}\right)=$ $A_{e}\left(E_{e} / E_{0}\right)^{-\alpha} \exp \left(-E_{e} / E_{\mathrm{e}, \mathrm{cut}}\right)$, where $E_{0}=0.5 \mathrm{GeV}$ is a reference energy. The cutoff energy $\left(E_{\mathrm{e}, \mathrm{cut}}\right)$, the injection spectral index $(\alpha)$, and the minimum electron energy $\left(E_{e, \min }\right)$ are found from modeling the synchrotron and IC spectrum, using the open-source code GAMERA ${ }^{1}$. It solves the transport equation to calculate the spectrum of particles $N_{e}\left(E_{e}, t\right)$ at a time $t$. We assume the electrons escape over dynamical timescale, $t_{\mathrm{esc}}^{e}=t_{\mathrm{dyn}}=R / c$, which is constant in energy and time.

\footnotetext{
${ }^{1}$ http://libgamera.github.io/GAMERA/docs/main_page.html
} 

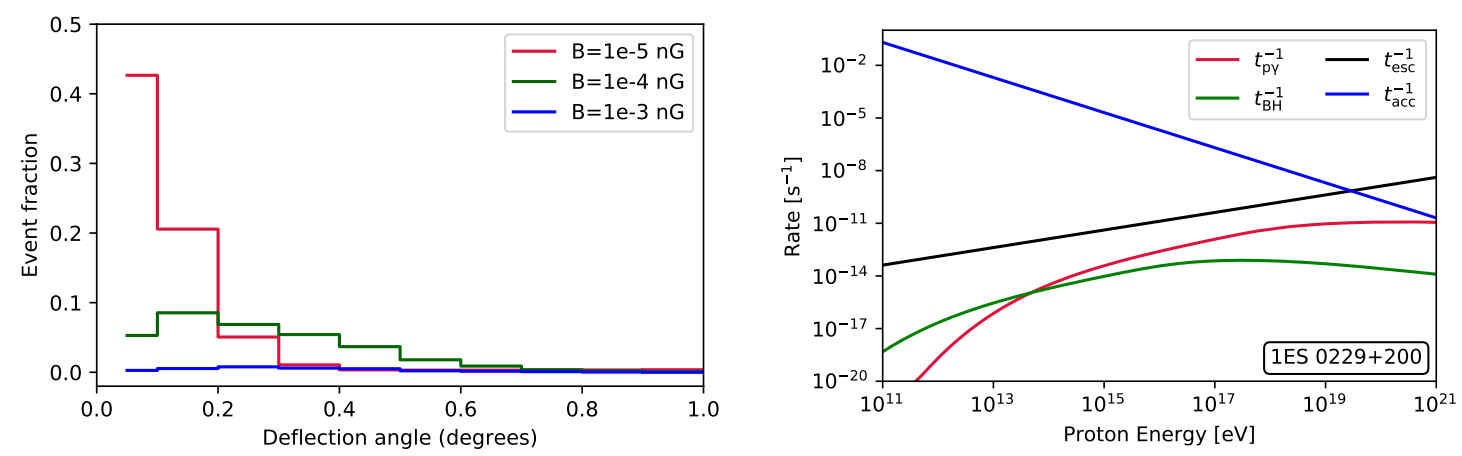

Figure 1: Left: Distribution of propagated UHECRs as a function of deflection angle in a random turbulent magnetic field. Right: Timescale of photohadronic interactions inside the jet with synchrotron and IC photons, along with the acceleration and escape timescale; for one of the sources considered in the study [8].

The photons from the jet are Doppler boosted $\delta_{D}=[\Gamma(1-\beta \cos \theta)]^{-1}$, where $\Gamma$ is the bulk Lorentz factor, and $\beta c$ is the velocity of the emitting plasma. $\theta \lesssim 1 / \Gamma$ is the viewing angle of the blazar with respect to the line of sight. The synchrotron and SSC luminosities are doppler boosted by $\delta_{D}^{4}$ in the observer frame [4]. The luminosity in electrons required in the AGN frame is $L_{\mathrm{e}}=\pi R^{2} \Gamma^{2} c u_{e}^{\prime}$, where $u_{e}^{\prime}$ is the energy density of electrons. The luminosity in magnetic field, i.e., the power carried as Poynting flux is given by $L_{\mathrm{B}}=R^{2} \Gamma^{2} \beta c B^{2} / 8$.

The maximum proton energies is given by the Hillas condition [5], $E_{\mathrm{p}, \max } \sim 2 \beta c Z e B R$. The proton injection is given by $N_{p}\left(E_{p}\right)=d N / d E_{p}=A_{p} E_{p}^{-\alpha}$, where we take $E_{\mathrm{p}, \min }=0.1 \mathrm{EeV}$ and $E_{\mathrm{p}, \max }=10 \mathrm{EeV}$. We calculate the timescales of photohadronic interactions on synchrotron and SSC photons inside the jet. Protons, being heavier than the electrons, are not cooled sufficiently inside the jet, and escape dominates over energy losses. The escape timescale of protons from the source is given as, $t_{\mathrm{esc}}^{p}=R^{2} / 4 D$, where we take the Kraichnan model of diffusion. The acceleration timescale is calculated from, $t_{\mathrm{acc}}^{p} \simeq 20 \eta r_{L} / 3 c \simeq 20 \eta \gamma_{p} m_{p} c / 3 e B$, where $\eta$ is the ratio of the mean magnetic field energy density to the turbulent magnetic field energy density [6]. We consider $\eta=1$ in our calculations. The escaping protons propagate through extragalactic distances interacting on CMB and EBL, producing electrons, positrons, $\gamma$-rays, and neutrinos. The secondary photons and electrons can initiate EM cascades resulting in photon spectrum down to $\mathrm{GeV}$ energies. The neutrinos, on the other hand, once produced propagate rectilinearly unhindered by EGMF or interactions. The proton luminosity required in the AGN frame is $L_{\mathrm{p}}=\pi R^{2} \Gamma^{2} c u_{p}^{\prime}$. We use the public code CRPropa 3 to propagate the UHECR protons from the source to observer [7] and DINT is used to propagate the EM particles produced from UHECR interactions. We consider the Gilmore et al. EBL model [9] and a random turbulent EGMF with a Kolmogorov power spectrum of RMS field strength $B_{\mathrm{rms}}=10^{-5} \mathrm{nG}$.

\section{Results}

We explore the effect of our choice of the extragalactic magnetic field (EGMF). We run 3D test simulations in CRPropa 3 for the propagation of UHECRs from a source at a distance of $1 \mathrm{Gpc}$ away from the observer to check the effect of varying $B_{\text {rms }}$. We consider a random turbulent magnetic 

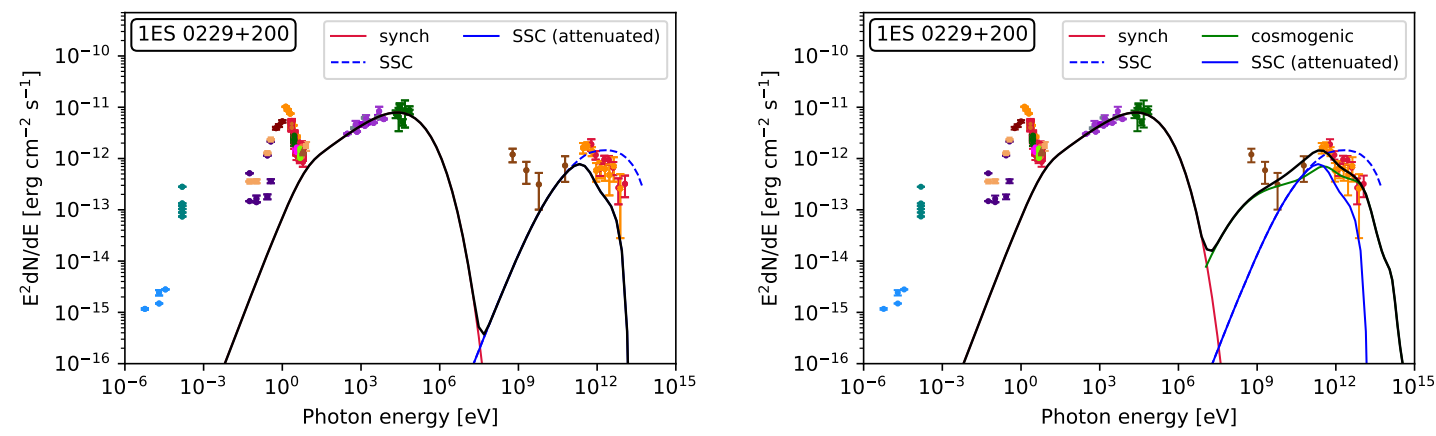

Figure 2: Multiwavelength spectrum of the HBLs, modeled using a pure leptonic model (left) and a leptonic + hadronic model (right). The figure is adaptd from Ref. [8].

field with Kolmogorov power spectrum and random polarization. In all cases, we set the value of the coherence length $l_{c}=1 \mathrm{Mpc}$ and thus take the observer to be a sphere of radius $R_{\mathrm{obs}}=1 \mathrm{Mpc}$. The proton injection spectrum is assumed to be a power law, $d N / d E_{p} \propto E^{-\alpha}$ with $\alpha=2$ and energies in the range $0.1-10 \mathrm{EeV}$. All energy loss processes, viz., pair production, photopion production, adiabatic losses, and beta decay, are included in the simulation. The deflections of the UHECRs on arrival at the surface of the observer sphere are calculated from initial and final momentum vector directions, shown in Fig. 1. The event fraction within $0-0.1^{\circ}$ indicates the survival rate $\xi_{B}$ of UHECRs close to the initial direction of propagation. We consider $B_{\mathrm{rms}}=10^{-5} \mathrm{nG}$ for the rest of our study to increase the fraction of events along the line-of-sight. Assuming the angle between the emission direction and line of sight to be a few degrees, the viewing angle $\theta \lesssim 1 / \Gamma$. Now, the flux of secondary cascade photons will be distributed across the area of the spherical cap subtended by the jet opening angle at the observer distance.

DINT solves the transport equation in 1D for the propagation and electromagnetic cascade of secondary $\mathrm{e}^{ \pm}$and $\gamma$ photons produced from UHECR interactions on CMB and EBL. Hence, we run 1D simulations in CRPropa to propagate UHECRs, producing EM particles, to calculate the secondary photon flux at the Earth. To find the flux intercepted by the observer's line of sight in the presence of an EGMF, we multiply this photon flux by $\xi_{B}$. Thus, the luminosity required in UHECR protons, $L_{\mathrm{UHECR}}$ can be calculated from the expression,

$$
L_{\mathrm{UHECR}}=\frac{2 \pi d_{L}^{2}\left(1-\cos \theta_{\mathrm{jet}}\right)}{\xi_{B} f_{\mathrm{CR}}} \int_{\epsilon_{\gamma, \min }}^{\epsilon_{\gamma, \max }} \epsilon_{\gamma} \frac{d N}{d \epsilon_{\gamma} d A d t} d \epsilon_{\gamma}
$$

where $2 \pi d_{L}^{2}\left(1-\cos \theta_{\text {jet }}\right)$ is the area of the spherical cap region. We consider typical values of $\theta_{\text {jet }} \sim 0.1$ radians $[11,12] . f_{\mathrm{CR}}$ is the ratio of the power in produced secondary photons $L_{\gamma, \mathrm{p}}$ due to the propagation of UHECRs and cascade of resulting EM particles to the injected UHECR power $L_{\text {UHECR }}$. Both $\xi_{B}$ and $f_{C R}$ will be a function of propagation distance. We obtain the secondary EM particles and cascade photons from 1D simulations and account for the conical distribution by calculating the luminosity according to Eqn. (1).

The observed spectrum is $F_{\gamma}^{\mathrm{obs}}(E, z)=F_{\gamma}^{\text {int }}(E, z) \exp \left[-\tau\left(E_{\gamma}, z_{0}\right)\right]$, where $\tau\left(E_{\gamma}, z\right)$ is the energy and redshift dependent optical depth of absorption in EBL. We consider the sources: 1ES 1011+496, 1ES 0229+200, 1ES 1101-232, and 1ES 0414+009. The timescale of photopion and Bethe-Heitler 


\begin{tabular}{cccccccccc}
\hline $\begin{array}{c}E_{\mathrm{e}, \min } \\
\mathrm{TeV}\end{array}$ & $\begin{array}{c}E_{\mathrm{e}, \text { cut }} \\
\mathrm{TeV}\end{array}$ & $\alpha$ & $\begin{array}{c}R \\
\mathrm{~cm}\end{array}$ & $\begin{array}{c}B \\
\text { Gauss }\end{array}$ & $\delta_{D}$ & $\begin{array}{c}L_{\mathrm{e}} \\
\mathrm{erg} / \mathrm{s}\end{array}$ & $\begin{array}{c}L_{\mathrm{B}} \\
\mathrm{erg} / \mathrm{s}\end{array}$ & $\begin{array}{c}L_{\mathrm{UHECR}} \\
\mathrm{erg} / \mathrm{s}\end{array}$ & $\begin{array}{c}L_{\mathrm{Edd}} \\
\mathrm{erg} / \mathrm{s}\end{array}$ \\
\hline \multicolumn{8}{c}{ Pure leptonic model } \\
0.01 & 1.5 & 2.2 & $1.0 \times 10^{16}$ & 0.015 & 40 & $1.3 \times 10^{38}$ & $1.3 \times 10^{41}$ & - & \\
\hline \multicolumn{8}{c}{ Leptonic + hadronic (UHECR) model } \\
0.01 & 1.5 & 2.2 & $1.0 \times 10^{16}$ & 0.015 & 40 & $1.3 \times 10^{38}$ & $1.3 \times 10^{41}$ & $2.6 \times 10^{43}$ & $1.7 \times 10^{47}$ \\
\hline
\end{tabular}

Table 1: Fit parameters for the multiwavelength SED modeling in Fig. 2. For detailed paramter values of all the sources considered in the study, please see Ref. [8].
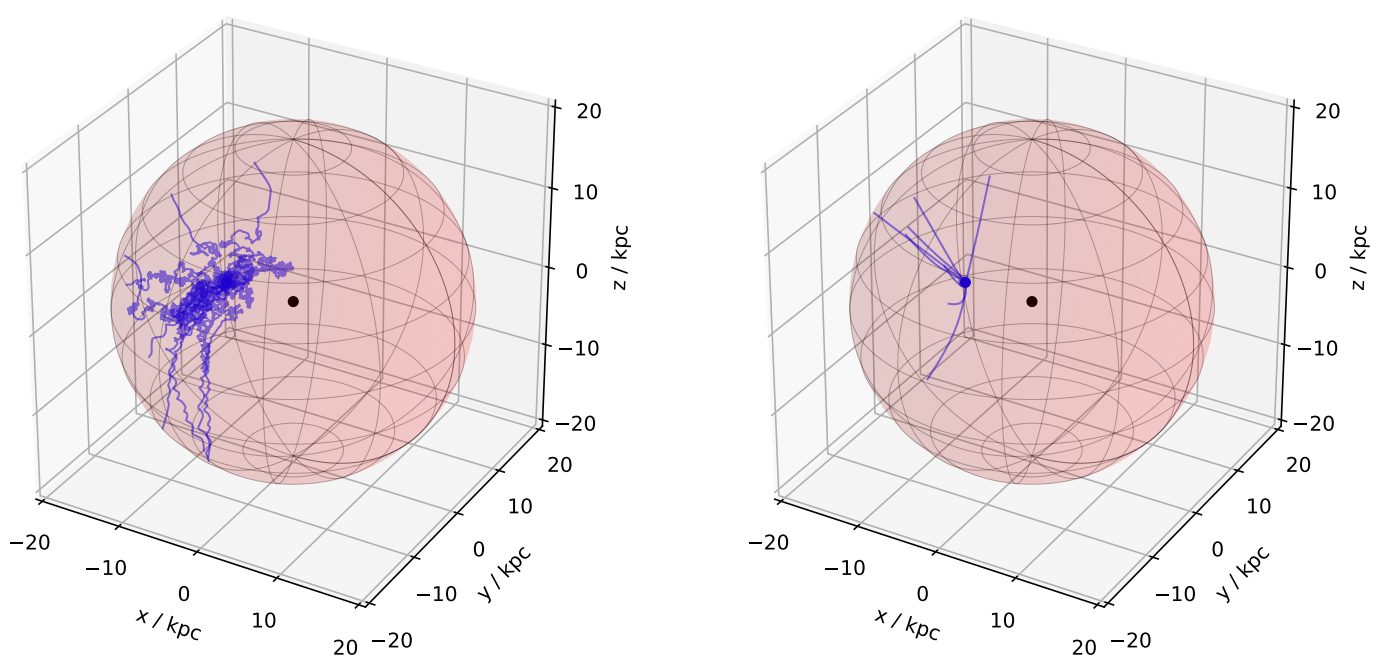

Figure 3: 3D trajectories of 10 UHECR anti-protons emitted isotropically and backtracked from the Earth in Janson \& Farrar Galactic magnetic field for $E=0.1 \mathrm{EeV}$ (left) and $E=10 \mathrm{EeV}$ (right). The black dot indicates the Galactic center. Figure adapted from Ref. [8].

interactions with synchrotron and IC photons inside the jet are compared with the escape timescale $t_{\text {esc }}$ and the acceleration timescale $t_{\text {acc }}$ (cf. Fig. 1). We consider the diffusion co-efficient $D_{0}$ adjusted between $10^{27}$ to $10^{30} \mathrm{~cm}^{2} / \mathrm{s}$, such that the acceleration rate dominates over escape rate at least up to $E=10^{19} \mathrm{eV}$. The latter is the $E_{\mathrm{p} \text {, max }}$ value considered in our simulations. The Eddington luminosity of the blazars is calculated using the expression $L_{\mathrm{Edd}}=10^{47}\left(M_{\mathrm{BH}} / 10^{9} M_{\odot}\right) \mathrm{erg} / \mathrm{s}$ [13], where $M_{\mathrm{BH}}$ is the mass of the central black hole. We assume $\delta_{D} \simeq \Gamma$ in our calculations, which is valid for a viewing angle of the order of few degrees. Here we present the results for only $1 \mathrm{ES}$ $0229+200$. Please refer to [8] for a detailed and fuller analysis.

The jet parameters obtained from fitting the synchrotron spectrum are used to calculate the SSC spectrum and are adjusted to extend to the highest energy possible. Beyond this energy, the hadronic contribution dominates. The photon spectrum produced in UHECR interactions, peaking at $\sim \mathrm{TeV}$ energies, is incorporated to explain the data points in the entire VHE range. The fit to photon SEDs obtained from pure leptonic and lepto-hadronic models are presented in the left and right panels of Fig. 2. The corresponding parameter values are given in the top section of Table 1. 


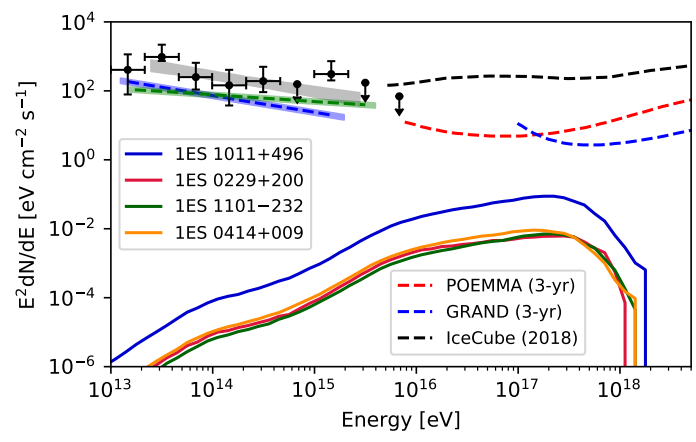

Figure 4: All-flavor neutrino flux at Earth produced in the same UHECR interactions as producing EM particles. The differential upper limit on extremely-high-energy cosmic neutrino flux by IceCube is also shown [15]. The black data points show the observed diffuse neutrino flux [16]. The flux upper limit from 2LAC blazars is shown using equal weighting for power-law with spectral index 2.5 (blue shaded region) and 2.2 (green shaded region) [17].

We have calculated the efficiency of $p \gamma$ interactions inside the jet using the formalism in [14] and found that the photon spectrum resulting from $\pi^{0}$ decay is insignificant in comparison to the observed flux. Thus, we consider the interactions of UHECR protons only during propagation over cosmological distances. The fit parameters are presented Table 1.

The Galactic magnetic field (GMF) creates a strong shadowing effect on the true location of the sources [18]. We do backtracking simulations of cosmic rays with the opposite charge, from the observer to the edge of the Galaxy, for showing UHECR trajectories in GMF. We consider the Jansson \& Farrar field model (JF12) [19]. For backtracking, 10 UHECR anti-protons are injected isotropically from the Earth and are similar to protons traveling towards Earth in forward simulations. We consider two such cases when the UHECR protons are observed at Earth with $E_{\mathrm{obs}}=0.1 \mathrm{EeV}$ and $10 \mathrm{EeV}$. The resulting trajectories are shown in the left and right panels of Fig. 3, respectively. The black and blue dots represent the Galactic center and the Earth at a distance of $8.5 \mathrm{kpc}$ from the Galactic center. Neutrinos are weakly interacting and hence travels undeflected by magnetic fields. The luminosity requirement in neutrinos can be written as $L_{v}=L_{\mathrm{UHECR}} \times f_{\mathrm{CR} \rightarrow v} \times \xi_{B}$, where $f_{\mathrm{CR} \rightarrow \nu}$ is the ratio of the power in produced secondary neutrinos $L_{\nu, p}$ due to propagation of UHECRs, to the injected UHECR power $L_{\text {UHECR }}$. The resulting all-flavor neutrino fluxes are orders of magnitude lower than the sensitivity of current and upcoming neutrino detectors (cf. Fig. 4).

\section{Discussions and Conclusions}

The one-zone leptonic emission model is often inadequate in explaining the high-energy $\gamma$-ray spectrum of some BL Lacs. These high-energy BL Lac objects (HBLs) exhibit a hard intrinsic $\mathrm{TeV}$ spectrum, which requires an alternate explanation. Among hadronic origin $\gamma$-ray models, those which invoke proton synchrotron emission require extremely high kinetic power and veryhigh values of doppler factor or magnetic field [20]. In this study, we consider UHECRs escape from the jet and interact with cosmic background photons (CMB and EBL) to produce secondary electromagnetic particles. These particles can initiate EM cascades leading to the production of 
VHE $\gamma$-ray spectrum. We find that the energy loss of protons inside the jet is much lower than the escape rate. For simplicity, we consider protons as the only UHECRs injected by these sources. We explain the multiwavelength SED of the HBLs by a suitable utilization of this lepto-hadronic model. The parameters for leptonic contribution are adjusted to extend the spectrum up to the highest energies possible and to fit the synchrotron spectrum simultaneously. Beyond this, the contribution from UHECR interactions dominates. The jet power required in such a scenario is calculated and compared with the Eddington luminosity. The total proton luminosity $L_{\mathrm{p}}$, considering relativistic protons down to $\sim 10 \mathrm{GeV}$ energies, will be approximately 5-10 times of the $L_{\text {UHECR }}$ value calculated. Taking this into account doesn't affect the credibility of our model, and the total jet power, in our case, remains lower than $L_{\mathrm{Edd}}$. The value of $\xi_{B}$ considered in the analysis is the survival rate of UHECRs within $0.1^{\circ}$ of the initial propagation direction. This factor is essential to constrict the propagation within a narrow cone leading to an increased probability of interception by the observer. If we calculate the survival rate of UHECRs within a smaller deflection angle than 0.1 degrees, a lower value of EGMF will be necessary.

Since the acceleration rate dominates over the escape rate up to $\gtrsim 10^{19} \mathrm{eV}$ and the maximum energy obtained from the Hillas condition comes out to be a few $\mathrm{EeV}$, we consider $E_{\mathrm{p} \text {, max }}=10^{19} \mathrm{eV}$ in our analysis. This also accounts for the uncertainty in fit parameters arising because electrons lose energy much faster than protons. As a result, they are restricted to a smaller region, while protons can travel larger distances without significant energy loss. Thus, the confinement regions of electrons and protons are most likely different. This can result in slightly different blob radii as viewed by electrons and protons. $E_{\mathrm{p} \text {,max }}$ is less than the threshold of photopion production on CMB, allowing the UHECR protons to travel cosmological distances and interact with EBL relatively close to Earth [2]. This justifies the competency of the UHECR interaction model, implemented in explaining the high-energy $\gamma$-ray spectrum from distant AGN. It is predicted in [21] that a hard intrinsic $\mathrm{TeV}$ spectrum of distant blazars showing no or little attenuation can be attributed to the fact that production of secondary $\gamma$-rays, occurring near to the observer compared to the source distance, dominates the observed $\gamma$-ray signal at the VHE regime. The flux of secondary neutrinos is inversely proportional to redshift, and the scaling is valid as long as the UHECR protons remain within the angular resolution of the detector [21]. The neutrino flux from individual BL Lacs obtained in our analysis is too low to be detected by currently operating and upcoming future detectors. Given the low maximum proton energy $(10 \mathrm{EeV})$ required in our model and deflections in the EGMF and GMF, identifying UHECRs coming from individual BL Lacs we discussed will be difficult.

\section{References}

[1] M. Boettcher, A. Reimer, K. Sweeney and A. Prakash, Astrophys. J. 768 (2013), 54 [arXiv:1304.0605].

[2] W. Essey and A. Kusenko, Astropart. Phys. 33 (2010), 81-85 [arXiv:0905.1162].

[3] S. Razzaque, C. D. Dermer and J. D. Finke, Astrophys. J. 745 (2012), 196 [arXiv:1110.0853].

[4] A. Celotti and G. Ghisellini, Mon. Not. Roy. Astron. Soc. 385 (2008), 283 [arXiv:0711.4112]. 
[5] A. M. Hillas, Ann. Rev. Astron. Astrophys. 22 (1984), 425-444

[6] P. O. Lagage, C. J. Cesarsky, Astron. Astrophys. 125 (1983), 249

[7] R. Alves Batista, A. Dundovic, M. Erdmann, K. H. Kampert, D. Kuempel, G. Müller, G. Sigl, A. van Vliet, D. Walz and T. Winchen, JCAP 05 (2016), 038 [arXiv:1603.07142].

[8] S. Das, N. Gupta and S. Razzaque, Astrophys. J. 889 (2020), 149 [arXiv:1911.06011].

[9] R. C. Gilmore, R. S. Somerville, J. R. Primack and A. Dominguez, Mon. Not. Roy. Astron. Soc. 422 (2012), 3189 [arXiv:1104.0671].

[10] S. Das, S. Razzaque and N. Gupta, Phys. Rev. D 99 (2019) no.8, 083015 [arXiv:1809.05321].

[11] A. B. Pushkarev, Y. Y. Kovalev, M. L. Lister and T. Savolainen, Astron. Astrophys. 507 (2009), L33 [arXiv:0910.1813].

[12] J. D. Finke, Astrophys. J. 870 (2019) no.1, 28 [arXiv:1811.04999].

[13] R. Falomo, N. Carangelo and A. Treves, Mon. Not. Roy. Astron. Soc. 343 (2003), 505 [arXiv:astro-ph/0304190].

[14] S. R. Kelner and F. A. Aharonian, Phys. Rev. D 78 (2008), 034013 [erratum: Phys. Rev. D 82 (2010), 099901] [arXiv:0803.0688].

[15] M. G. Aartsen et al. (IceCube Collaboration), Phys. Rev. D 98 (2018) no.6, 062003 [arXiv:1807.01820].

[16] M. G. Aartsen et al. (IceCube Collaboration), Astrophys. J. 809 (2015) no.1, 98 [arXiv:1507.03991].

[17] M. G. Aartsen et al. (IceCube Collaboration), Astrophys. J. 835 (2017) no.1, 45 [arXiv:1611.03874].

[18] N. Fraija, E. Aguilar-Ruiz, A. Galván-Gámez, A. Marinelli and J. A. de Diego, Mon. Not. Roy. Astron. Soc. 481 (2018) no.4, 4461-4471 [arXiv:1709.05766].

[19] R. Jansson and G. R. Farrar, Astrophys. J. 757 (2012), 14 [arXiv:1204.3662].

[20] M. Petropoulou and A. Mastichiadis, Mon. Not. Roy. Astron. Soc. 426 (2012), 462-472 [arXiv:1207.5227].

[21] W. Essey, O. Kalashev, A. Kusenko and J. F. Beacom, Astrophys. J. 731 (2011), 51 [arXiv:1011.6340]. 